\title{
Exact Constructions of a Family of Dense Periodic Packings of Tetrahedra
}

\author{
S. Torquato ${ }^{1,2,3,4,5,6}$ and Y. Jiao ${ }^{5}$ \\ ${ }^{1}$ Department of Chemistry, Princeton University, \\ Princeton New Jersey 08544, USA \\ ${ }^{2}$ Department of Physics, Princeton University, \\ Princeton New Jersey 08544, USA \\ ${ }^{3}$ Princeton Center for Theoretical Science, \\ Princeton University, Princeton New Jersey 08544, USA \\ ${ }^{4}$ Program in Applied and Computational Mathematics, \\ Princeton University, Princeton New Jersey 08544, USA \\ ${ }^{5}$ School of Natural Sciences, Institute for Advanced Study, Princeton NJ 08540 and \\ ${ }^{6}$ Department of Mechanical and Aerospace Engineering, \\ Princeton University, Princeton New Jersey 08544, USA
}

\begin{abstract}
The determination of the densest packings of regular tetrahedra (one of the five Platonic solids) is attracting great attention as evidenced by the rapid pace at which packing records are being broken and the fascinating packing structures that have emerged. Here we provide the most general analytical formulation to date to construct dense periodic packings of tetrahedra with four particles per fundamental cell. This analysis results in six-parameter family of dense tetrahedron packings that includes as special cases recently discovered "dimer" packings of tetrahedra, including the densest known packings with density $\phi=\frac{4000}{4671}=0.856347 \ldots$ This study strongly suggests that the latter set of packings are the densest among all packings with a four-particle basis. Whether they are the densest packings of tetrahedra among all packings is an open question, but we offer remarks about this issue. Moreover, we describe a procedure that provides estimates of upper bounds on the maximal density of tetrahedron packings, which could aid in assessing the packing efficiency of candidate dense packings.
\end{abstract}

PACS numbers: $61.50 . \mathrm{Ah}, 05.20 . \mathrm{Jj}$ 


\section{INTRODUCTION}

Dense packings of nonoverlapping solid objects or particles are ubiquitous in synthetic and natural situations. Packing problems arise in technological contexts, such as the packaging industries, agriculture (e.g., grains in silos), and solid-rocket propellants, and underlie the structure of a multitude of biological systems (e.g., tissue structure, cell membranes, and phyllotaxis). Dense particle packings are intimately related to the structure of lowtemperature states of condensed matter, such as liquids, glasses and crystals [1-4]. In the last decade, scientific attention has broadened from the study of dense packings of spheres (the simplest shape that does not tile Euclidean space) [5-14] to dense packings of disordered [15-19] and ordered [20 22] nonspherical particles. In addition, the equilibrium phase behavior and transport properties of hard nonspherical particles have been of topics of great interest [23-26]. Nonsphericity introduces rotational degrees of freedom not present in sphere packings, and can dramatically alter the characteristics from those of sphere packings.

A basic characteristic of a packing in $d$-dimensional Euclidean space $\mathbb{R}^{d}$ is its density $\phi$, defined to be the fraction of $\mathbb{R}^{d}$ that is covered by the particles. A problem that has been of great scientific interest for centuries is the determination of the densest arrangement(s) of particles that do not tile space and the associated maximal density $\phi_{\max }$. For generally shaped particles, finding the densest packings is notoriously difficult. This salient point is summarized well by Henry Cohn [27] who recently remarked, "For most grain shapes we cannot guess or even closely approximate the answer, let alone prove it, and it is difficult to develop even a qualitative understanding of the effects of grain shape on packing density." Until recently, very little was known about the densest packings of polyhedral particles. The difficulty in obtaining dense packings of polyhedra is related to their complex rotational degrees of freedom and to the non-smooth nature of their shapes [28, 29].

Recently, we set out to attempt to determine the densest known packings of the Platonic

and Archimedean solids [28, 29]. It was shown that the central symmetry of the majority of the Platonic and Archimedean solids distinguish their dense packing arrangements from those of the non-centrally symmetric ones in a fundamental way. (A particle is centrally symmetric if it has a center $C$ that bisects every chord through $C$ connecting any two boundary points of the particle; i.e., the center is a point of inversion symmetry.) The tetrahedron is the only Platonic solid that lacks central symmetry, an attribute that geometrically frus- 
trates it to a greater degree than the majority of the remaining solids in this set that do not tile space [29]. A number of organizing principles emerged in the form of conjectures for polyhedra as well as other nonspherical shapes. In the case of polyhedra, the following three are particularly relevant and will be applied later in the paper to remark on the optimality of tetrahedron packings:

- Conjecture 1: The densest packings of the centrally symmetric Platonic and Archimedean solids are given by their corresponding optimal Bravais lattice packings.

- Conjecture 2: The densest packing of any convex, congruent polyhedron without central symmetry generally is not a Bravais lattice packing, i.e., the set of such polyhedra whose optimal packing is not a Bravais lattice is overwhelmingly larger than the set whose optimal packing is a Bravais lattice.

- Conjecture 3: The densest packings of congruent, centrally symmetric particles that do not possesses three equivalent principle axes (e.g., ellipsoids) generally cannot be Bravais lattices.

Conjecture 1 is the analog of Kepler's sphere conjecture for the centrally symmetric Platonic and Archimedean solids. In this sense, such solids behave similarly to spheres in that their densest packings are lattice arrangements and (except for the cube and the truncated octahedron) are geometrically frustrated like spheres. Conjecture 2 has been shown by Conway and Torquato [30] to be true for both the tetrahedron and Archimedean non-centrally symmetric truncated tetrahedron, the latter of which can be arranged in a "uniform" non-Bravais lattice packing with density at least as high as $\frac{23}{24}=0.958333 \ldots$... (A uniform packing has a symmetry operation, in this case the point inversion symmetry, that takes any particle into another.) Although Conjecture 3 is explicitly stated in its current form here for the first time, it was strongly implied in Ref. [29].

It was Conway and Torquato's investigation [30] that has spurred the flurry of activity in the last several years to find the densest packings of tetrahedra. There have been many twists and unexpected turns since 2006 that have led to the dense packings of tetrahedra that we report here. Therefore, to place our present results in their proper context, it is instructive to review briefly the developments since 2006. 
TABLE I: A brief summary of the dense non-lattice packings of tetrahedra. The name of the packing is given along with the year that it was discovered. Here $\phi$ is the packing density and $N$ is the number of tetrahedra per fundamental cell.

\begin{tabular}{cccc}
\hline \hline Packing & Year & $\phi$ & $N$ \\
\hline Uniform I [30] & 2006 & $\frac{2}{3}=0.666666 \ldots$ & 2 \\
Welsh [30] & 2006 & $\frac{17}{24}=0.708333 \ldots$ & 34 \\
Icosahedral [30] & 2006 & $0.716559 \ldots$ & 20 \\
Uniform II [35] & 2009 & $\frac{139+40 \sqrt{10}}{369}=0.719488 \ldots$ & 2 \\
Wagon Wheels [33] & 2008 & $0.778615 \ldots$ & 18 \\
Improved Wagon Wheels [28] & 2009 & $0.782021 \ldots$ & 72 \\
Disordered Wagon Wheels [29] & 2009 & $0.822637 \ldots$ & 314 \\
Ring Stacks [34] & 2009 & $0.8503 \ldots$ & 82 \\
Uniform III [35] & 2009 & $\frac{100}{117}=0.854700 \ldots$ & 4 \\
Dimer-Uniform I [36] & 2009 & $\frac{12250}{14319}=0.855506 \ldots$ & 4 \\
Dimer-Uniform II [37] & 2010 & $\frac{4000}{4671}=0.856347 \ldots$ & 4 \\
\hline \hline
\end{tabular}

First, we note that the densest Bravais-lattice packing of tetrahedra (requiring one tetrahedron per fundamental cell such that each tetrahedron in the packing has the same orientation as the others) has a packing fraction $\phi=\frac{18}{49}=0.367 \ldots$ and each tetrahedron touches 14 others [31]. Conway and Torquato [30] showed that the densest packings of tetrahedra cannot be Bravais lattices by analytically constructing several such packings with densities that are substantially larger than $\frac{18}{49}$. (A non-Bravais lattice packing contains multiple particles, with generally different orientations, per fundamental cell, which is periodically replicated in $\mathbb{R}^{d}$.) One such packing is a "uniform" packing with density $\phi=\frac{2}{3}$ and two particles per fundamental cell. The so-called "Welsh" packing has a density $\phi=0.708333 \ldots$ and 34 particles per fundamental cell. Yet another non-Bravais lattice packing with density $\phi=0.716559 \ldots$ is based on the filling of "imaginary" icosahedra with the densest arrangement of 20 tetrahedra and then arranging the imaginary icosahedra in their densest lattice packing configuration. The densities of both the Welsh and Icosahedral packings can be further improved by certain particle displacements [30]. Using imperfect "tetrahedral" dice, Chaikin et al. [32] experimentally generated jammed disordered packings with 
$\phi \approx 0.75$. Employing physical models and a computer algebra system, Chen [33] discovered a remarkably dense periodic arrangement of tetrahedra with $\phi=0.7786 \ldots$, which exceeds the density $\left(\phi_{\max }=\pi / \sqrt{18}=0.7404 \ldots\right)$ of the densest sphere packing by an appreciable amount. We have called this the "wagon-wheels" packing [28, 29].

Torquato and Jiao [28] devised and applied an optimization scheme, called the adaptiveshrinking-cell (ASC) method, that used an initial configuration based on the wagon-wheels packing to yield a non-Bravais lattice packing consisting of 72 tetrahedra per fundamental cell with a density $\phi=0.782 \ldots$ [28]. Using 314 particles per fundamental cell and starting from an "equilibrated" low-density liquid configuration, the same authors were able to improve the density to $\phi=0.823 \ldots$. [29]. This packing arrangement interestingly lacks longrange order. Haji-Akbari et al. [34] numerically constructed a periodic packing of tetrahedra made of parallel stacks of "rings" around "pentagonal" dipyramids consisting of 82 particles per fundamental cell and a density $\phi=0.8503 \ldots$... More recently, Kallus et al. [35] found a remarkably simple uniform packing of tetrahedra with high symmetry consisting of only four particles per fundamental cell with density $\phi=\frac{100}{117}=0.854700 \ldots$. We subsequently presented an analytical formulation to construct dense uniform dimer packings of tetrahedra and employed it to obtain a three-parameter family of packings. (A dimer is composed of a pair of regular tetrahedra that exactly share a common face. A uniform dimer packing of tetrahedra takes any dimer via a point-inversion symmetry operation into another.) Making an assumption about one of these parameters resulted in a two-parameter family, including those with density as high as $\phi=\frac{12250}{14319}=0.855506 \ldots$ [36]. Chen et al. [37] recognized that we made such an assumption and employed a similar formalism to obtain a threeparameter family of tetrahedron packings, including the densest known dimer packings of tetrahedra with a density $\phi=\frac{4000}{4671}=0.856347 \ldots$. Table $\$ summarizes some of the packing characteristics of the non-Bravais lattice packings of tetrahedra.

In the following section, we provide the details of our more general formulation and construct a six-parameter family of dense tetrahedron packings. We will show that our formalism includes as special cases all of the recently discovered four-particle basis packings [35 37]. Our analysis strongly suggests that the optima among this set of packings provide the densest arrangements among all packings with a four-particle basis. In Sec. III, we describe a procedure that provides estimates of upper bounds on the maximal density of tetrahedron packings, which could aid in assessing the packing efficiency of candidate dense 
packings. In Sec. IV, we make concluding remarks, including comments on the optimality of the densest known dimer packings of tetrahedra.

\section{ANALYTICAL CONSTRUCTIONS OF DENSE PACKINGS OF TETRAHE- DRA}

Inspired by the work of Kallus et al. [35], we have applied the adaptive-shrinking-cell (ASC) optimization scheme to examine comprehensively packings with a considerably small number of particles per fundamental cell (from 2 to 32) than we have used in the past [28, 29]. The ASC scheme employs both a sequential search of the configurational space of the particles and the space of lattices via an adaptive fundamental cell that deforms and shrinks on average to obtain dense packings. A dense packing with 8-particle basis that emerged from this numerical investigation suggested that it was composed of two very similar fundamental cells, each containing 4 particles. Using one of the 4-particle basis configurations, we were able to find packings with density $\phi=0.8551034 \ldots$ that exceeded the highest density packings with $\phi=\frac{100}{117}=0.854708 \ldots$ constructed by Kallus et al. Even though our packings possess a type of point inversion symmetry, they are not as symmetric as the densest packings reported in Ref. [35], as we now explain.

The four tetrahedra in the fundamental cell in our dense numerically generated packings formed two contacting "dimers". A dimer is composed of a pair of regular tetrahedra with unit edge length that exactly share a common face. The compound object consisting of the two contacting dimers possesses point inversion symmetry, with the inversion center at the centroid of the contacting region on the faces. A Bravais lattice possesses point inversion symmetry about the lattice points and the centroids of the fundamental cells. By placing the symmetry center of the two-dimer compound on the centroids (or the lattice points), we construct packings that generally possess point inversion symmetry only about the symmetry centers of the two-dimer compound. Besides the centroids of the fundamental cell, all of the half-integer lattice points are also inversion symmetry centers of the packing. We call such structures dimer-uniform packings, since the inversion symmetry acts to take any dimer into another. Such packings should be distinguished from the more symmetric uniform (or transitive) packings of tetrahedra in which the symmetry operation acts to take any tetrahedron into another, such as the ones found in Refs. [30] and [35] (see Table [I). 
The latter have almost as much symmetry as a Bravais lattice, except that the centroids of the particles are not just characterized by simple translational symmetry.

\section{A. General Formalism: Six-Parameter Family of Dense Tetrahedron Packings}

We now describe our general analytical formulation to construct dense tetrahedron packings by relaxing the symmetry conditions on the contacting dimers in detail. In particular, we orient the 3-fold rotational symmetry axis of one of the dimers in an arbitrary direction (say the $z$-direction of a Cartesian coordinate system), and then fix the origin of the lattice vectors at the centroid of this dimer. (The centroid is located at the center of the contacting faces of the two tetrahedra that comprise the dimer.) Then we place the second dimer in contact with the first one such that there is a center of inversion symmetry that takes one dimer to the other, which implies a face-to-face contact between the two dimers.

The problem of determining the analytical constructions then amounts to determining 12 equations for the 12 unknowns. Nine of the 12 unknowns arise from the three unknown lattice vectors, each of which contains three unknown components. The other 3 unknowns derive from the components of the centroid of the second dimer.

In particular, we let the centroid of the dimer at the origin be denoted by $\mathbf{c}_{0}=(0,0,0)$, and the centroid of the other dimer be denoted by $\mathbf{c}_{1}=\left(\eta_{1}, \eta_{2}, \eta_{3}\right)$. The vertices of the two dimers associated with $\mathbf{c}_{0}$ and $\mathbf{c}_{1}$ are given by $\mathbf{v}_{A}=\left(\frac{1}{2}, \frac{1}{2 \sqrt{3}}, 0\right), \mathbf{v}_{B}=\left(-\frac{1}{2}, \frac{1}{2 \sqrt{3}}, 0\right)$, $\mathbf{v}_{C}=\left(0,-\frac{1}{\sqrt{3}}, 0\right), \mathbf{v}_{D}=\left(0,0, \sqrt{\frac{2}{3}}\right), \mathbf{v}_{E}=\left(0,0,-\sqrt{\frac{2}{3}}\right)$ and $\mathbf{v}_{A}^{*}=-\mathbf{v}_{A}+\mathbf{c}_{1}, \mathbf{v}_{B}^{*}=-\mathbf{v}_{B}+\mathbf{c}_{1}$, $\mathbf{v}_{C}^{*}=-\mathbf{v}_{C}+\mathbf{c}_{1}, \mathbf{v}_{D}^{*}=-\mathbf{v}_{D}+\mathbf{c}_{1}, \mathbf{v}_{E}^{*}=-\mathbf{v}_{E}+\mathbf{c}_{1}$, respectively. In addition, let the lattice vectors be $\boldsymbol{\lambda}_{1}=\left(-\alpha_{1},-\alpha_{2},-\alpha_{3}\right), \boldsymbol{\lambda}_{2}=\left(\beta_{1},-\beta_{2},-\beta_{3}\right)$, and $\boldsymbol{\lambda}_{3}=\left(\gamma_{1}, \gamma_{2}, \gamma_{3}\right)$. The 12 components of the four vectors $\mathbf{c}_{1}$ and $\boldsymbol{\lambda}_{i},(i=1,2,3)$ are the variables that determine the packing. Note in the above general set-up, we assume no particular symmetry of the packings.

In our packings, the dimers have 8 possible face-to-face contacts, 6 possible edge-to-edge contacts and 4 possible vertex-to-face contacts. Each contact provides a condition that either reduces the number of variables for the packing or constrains their feasible values.

A face-to-face contact requires that the projection of the vector distance between the centroids of the two dimers on the contacting face normal is equal to the diameter of the insphere (the largest possible sphere that can be inscribed into a tetrahedron) of the tetrahedra. A 


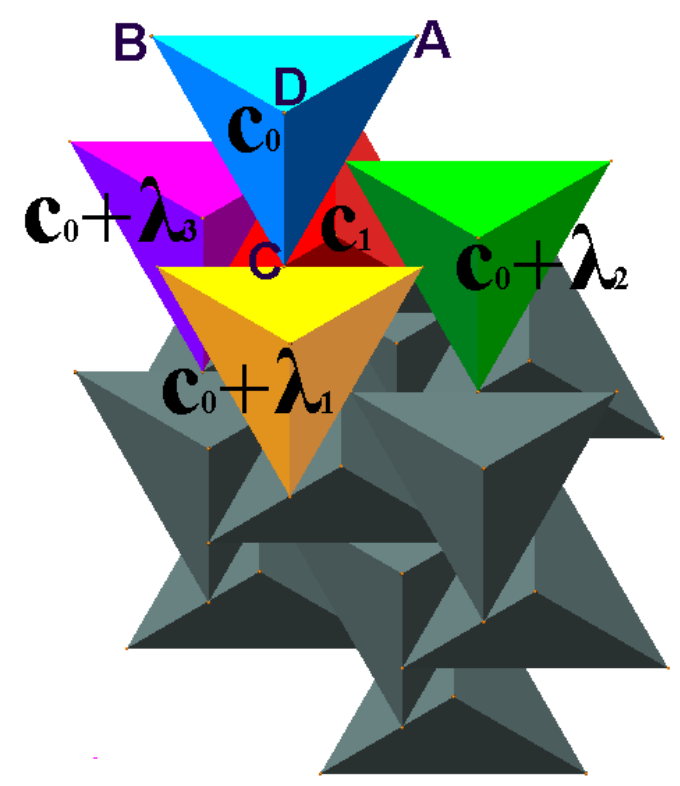

FIG. 1: (color online). The geometrical set up for the general formulation of the analytical constructions of dimer packings of tetrahedra. The dimer (two tetrahedra sharing a common face) centered at $\mathbf{c}_{0}$ is shown in blue with the vertices labeled (note that $\mathbf{v}_{E}$ whose projection coincides with $\mathbf{v}_{D}$ is not shown). The dimer centered at $\mathbf{c}_{1}$ is shown in red. The dimer centered at $\left(\mathbf{c}_{0}+\boldsymbol{\lambda}_{1}\right)$ is shown in yellow. The dimer centered at $\left(\mathbf{c}_{0}+\boldsymbol{\lambda}_{2}\right)$ is shown in green. The dimer centered at $\left(\mathbf{c}_{0}+\boldsymbol{\lambda}_{3}\right)$ is shown in purple. Observe that in the perspective shown the dimers appear as if they were single tetrahedra.

nonoverlapping condition associated with a face-to-face contact requires that the projection of the vector distance between the centroids of the two dimers on the contacting face normal is greater than the diameter of the insphere. The 8 possible face-to-face contacts are between the dimer pairs with the centroids at $\left\{\mathbf{c}_{1}, \mathbf{c}_{0}\right\},\left\{\mathbf{c}_{1}, \mathbf{c}_{0}+\boldsymbol{\lambda}_{1}\right\},\left\{\mathbf{c}_{1}, \mathbf{c}_{0}+\boldsymbol{\lambda}_{2}\right\},\left\{\mathbf{c}_{0}+\boldsymbol{\lambda}_{3}, \mathbf{c}_{1}\right\}$, $\left\{\mathbf{c}_{0}+\boldsymbol{\lambda}_{3}, \mathbf{c}_{1}-\boldsymbol{\lambda}_{2}\right\},\left\{\mathbf{c}_{0}+\boldsymbol{\lambda}_{3}, \mathbf{c}_{1}+\boldsymbol{\lambda}_{1}-\boldsymbol{\lambda}_{2}\right\},\left\{\mathbf{c}_{0}, \mathbf{c}_{1}-\boldsymbol{\lambda}_{1}+\boldsymbol{\lambda}_{3}\right\}$, and $\left\{\mathbf{c}_{0}, \mathbf{c}_{1}+\boldsymbol{\lambda}_{1}-\boldsymbol{\lambda}_{2}-2 \boldsymbol{\lambda}_{3}\right\}$. The possible contact between dimer pairs at $\left\{\mathbf{u}_{i}, \mathbf{u}_{j}\right\}$ requires

$$
\left(\mathbf{u}_{i}-\mathbf{u}_{j}\right) \cdot \mathbf{n}_{i j} \geq \frac{2 \sqrt{6}}{9}
$$

where $\mathbf{n}_{i j}$ is unit outward contacting face normal of the dimer at $\mathbf{u}_{j}$. In the following discussion, we explicitly provide these equations.

First, we note that $\mathbf{c}_{1}$ can be completely determined by considering the contacts that 
the dimer associated with it makes with three other dimers centered at $\mathbf{c}_{0},\left(\mathbf{c}_{0}+\boldsymbol{\lambda}_{1}\right)$ and $\left(\mathbf{c}_{0}+\boldsymbol{\lambda}_{2}\right)$ (see Fig. 1). Note that $\boldsymbol{\lambda}_{1}$ and $\boldsymbol{\lambda}_{2}$ are two of the lattice vectors. The equations associated with these three contacts are respectively given by

$$
\begin{gathered}
\frac{1}{6} \eta_{1}-\frac{1}{6 \sqrt{3}} \eta_{2}-\frac{\sqrt{2}}{12 \sqrt{3}} \eta_{3}=\frac{1}{9} \\
\frac{1}{3 \sqrt{3}}\left(\eta_{2}+\alpha_{2}\right)-\frac{\sqrt{2}}{12 \sqrt{3}}\left(\eta_{3}+\alpha_{3}\right)=\frac{1}{9}, \\
-\frac{1}{6}\left(\eta_{1}-\beta_{1}\right)-\frac{1}{6 \sqrt{3}}\left(\eta_{2}+\beta_{2}\right)-\frac{\sqrt{2}}{12 \sqrt{3}}\left(\eta_{3}+\beta_{3}\right)=\frac{1}{9} .
\end{gathered}
$$

By solving Eqs. (2) - (4), we can obtain the components $\left(\eta_{1}, \eta_{2}, \eta_{3}\right)$ of $\mathbf{c}_{1}$, i.e.,

$$
\begin{gathered}
\eta_{1}=\frac{1}{12}\left(6 \beta_{1}-2 \sqrt{3} \beta_{2}-\sqrt{6} \beta_{3}\right), \\
\eta_{2}=\frac{1}{12}\left(-8 \alpha_{2}+2 \sqrt{2} \alpha_{3}+2 \sqrt{3} \beta_{1}-2 \beta_{2}-\sqrt{2} \beta_{3}\right), \\
\eta_{3}=-\frac{1}{3 \sqrt{2}}\left(4 \sqrt{3}-4 \alpha_{2}+\sqrt{2} \alpha_{3}-2 \sqrt{3} \beta_{1}+2 \beta_{2}+\sqrt{2} \beta_{3}\right),
\end{gathered}
$$

Moreover, we note that $\boldsymbol{\lambda}_{3}$ can be completely determined by considering the contacts between the dimer centered at $\left(\mathbf{c}_{0}+\boldsymbol{\lambda}_{3}\right)$ and at $\mathbf{c}_{1},\left(\mathbf{c}_{1}-\boldsymbol{\lambda}_{2}\right)$ and $\left(\mathbf{c}_{1}+\boldsymbol{\lambda}_{1}-\boldsymbol{\lambda}_{2}\right)$, respectively. The equations associated with these three contacts are given by

$$
\begin{gathered}
\frac{1}{6}\left(\eta_{1}-\gamma_{1}\right)-\frac{1}{6 \sqrt{3}}\left(\eta_{2}-\gamma_{2}\right)+\frac{\sqrt{2}}{12 \sqrt{3}}\left(\eta_{3}-\gamma_{3}\right)=\frac{1}{9} . \\
\frac{1}{3 \sqrt{3}}\left(\eta_{2}+\beta_{2}-\gamma_{2}\right)+\frac{\sqrt{2}}{12 \sqrt{3}}\left(\eta_{3}+\beta_{3}-\gamma_{3}\right)=\frac{1}{9} \\
-\frac{1}{6}\left(\eta_{1}+\alpha_{1}-\beta_{1}-\gamma_{1}\right)-\frac{1}{6 \sqrt{3}}\left(\eta_{2}+\alpha_{2}+\beta_{2}-\gamma_{2}\right)+\frac{\sqrt{2}}{12 \sqrt{3}}\left(\eta_{3}+\alpha_{3}+\beta_{3}-\gamma_{3}\right)=\frac{1}{9} .
\end{gathered}
$$

By solving Eqs. (8) - (10), we can obtain the components $\left(\gamma_{1}, \gamma_{2}, \gamma_{3}\right)$ of $\boldsymbol{\lambda}_{3}$, i.e.,

$$
\begin{aligned}
& \gamma_{1}=\frac{1}{12}\left(-6 \alpha_{1}-2 \sqrt{3} \alpha_{2}+\sqrt{6} \alpha_{3}-2 \sqrt{6} \beta_{3}\right), \\
& \gamma_{2}=\frac{1}{12}\left(-2 \sqrt{3} \alpha_{1}-10 \alpha_{2}+3 \sqrt{2} \alpha_{3}+8 \beta_{2}\right),
\end{aligned}
$$




$$
\gamma_{3}=\frac{1}{3}\left(-4 \sqrt{6}+\sqrt{6} \alpha_{1}+3 \sqrt{2} \alpha_{2}-2 \alpha_{3}+2 \sqrt{6} \beta_{1}+\beta_{3}\right) .
$$

By achieving the aforementioned 6 face-to-face contacts, we can reduce the number of independent variables for the packing from 12 to 6 , with the remaining variables being $\alpha_{i}$ and $\beta_{i}(i=1,2,3)$. In other words, all of the lattice vectors and the centroids of dimers are completely specified by the six parameters $\alpha_{i}$ and $\beta_{i}$, which gives the six-parameter family of dense packings, i.e.,

$$
\begin{gathered}
\mathbf{c}_{1}=\left[\begin{array}{c}
\frac{1}{12}\left(6 \beta_{1}-2 \sqrt{3} \beta_{2}-\sqrt{6} \beta_{3}\right) \\
\frac{1}{12}\left(-8 \alpha_{2}+2 \sqrt{2} \alpha_{3}+2 \sqrt{3} \beta_{1}-2 \beta_{2}-\sqrt{2} \beta_{3}\right) \\
-\frac{1}{3 \sqrt{2}}\left(4 \sqrt{3}-4 \alpha_{2}+\sqrt{2} \alpha_{3}-2 \sqrt{3} \beta_{1}+2 \beta_{2}+\sqrt{2} \beta_{3}\right)
\end{array}\right]^{T}, \\
\boldsymbol{\lambda}_{1}=\left(\begin{array}{cc}
\left.-\alpha_{1}, \quad-\alpha_{2}, \quad-\alpha_{3}\right), \quad \boldsymbol{\lambda}_{2}=\left(\beta_{1}, \quad-\beta_{2}, \quad-\beta_{3}\right), \\
\boldsymbol{\lambda}_{3}=\left[\begin{array}{c}
\frac{1}{12}\left(-6 \alpha_{1}-2 \sqrt{3} \alpha_{2}+\sqrt{6} \alpha_{3}-2 \sqrt{6} \beta_{3}\right) \\
\frac{1}{12}\left(-2 \sqrt{3} \alpha_{1}-10 \alpha_{2}+3 \sqrt{2} \alpha_{3}+8 \beta_{2}\right) \\
\frac{1}{3}\left(-4 \sqrt{6}+\sqrt{6} \alpha_{1}+3 \sqrt{2} \alpha_{2}-2 \alpha_{3}+2 \sqrt{6} \beta_{1}+\beta_{3}\right)
\end{array}\right]^{T} .
\end{array}\right.
\end{gathered}
$$

Note that these parameters can not be varied completely independently of each other, i.e., they are related by the additional nonoverlapping conditions which will be given in the ensuing discussions.

There are two additional possible face-to-face contacts between dimer pairs centered at $\left\{\mathbf{c}_{0}, \mathbf{c}_{1}-\boldsymbol{\lambda}_{1}+\boldsymbol{\lambda}_{3}\right\}$, and $\left\{\mathbf{c}_{0}, \mathbf{c}_{1}+\boldsymbol{\lambda}_{1}-\boldsymbol{\lambda}_{2}-2 \boldsymbol{\lambda}_{3}\right\}$. The equations associated with these possible contacts are given by

$$
\begin{gathered}
-\frac{1}{6}\left(\eta_{1}+\alpha_{1}+\gamma_{1}\right)-\frac{1}{6 \sqrt{3}}\left(\eta_{2}+\alpha_{2}+\gamma_{2}\right)-\frac{\sqrt{2}}{12 \sqrt{3}}\left(\eta_{3}+\alpha_{3}+\gamma_{3}\right) \geq \frac{1}{9}, \\
-\frac{1}{6}\left(\eta_{1}-\alpha_{1}-\beta_{1}-2 \gamma_{1}\right)+\frac{1}{6 \sqrt{3}}\left(\eta_{2}-\alpha_{2}+\beta_{2}-2 \gamma_{2}\right)-\frac{\sqrt{2}}{12 \sqrt{3}}\left(\eta_{3}-\alpha_{3}+\beta_{3}-2 \gamma_{3}\right) \geq \frac{1}{9} .
\end{gathered}
$$

These conditions constrain the possible values of $\alpha_{i}$ and $\beta_{i}$.

An edge-to-edge contact requires that the projection of the vector connecting the corresponding ends of two edges on the common perpendicular line of the two edges equals zero. 
A nonoverlapping condition associated with an edge-to-edge contact requires that the projection of the two edges on the common perpendicular line of the two edges are completely separated. The 6 possible edge-to-edge contacts lead to 3 independent conditions, i.e.,

$$
\begin{gathered}
{\left[\mathbf{v}_{A}-\left(\mathbf{v}_{B}-\boldsymbol{\lambda}_{1}+\boldsymbol{\lambda}_{2}+\boldsymbol{\lambda}_{3}\right)\right] \cdot \mathbf{l}_{0} \geq 0} \\
{\left[\mathbf{v}_{C}-\left(\mathbf{v}_{B}-\boldsymbol{\lambda}_{1}+\boldsymbol{\lambda}_{3}\right)\right] \cdot \mathbf{l}_{1} \geq 0} \\
{\left[\mathbf{v}_{C}-\left(\mathbf{v}_{D}+\boldsymbol{\lambda}_{3}\right)\right] \cdot \mathbf{l}_{2} \geq 0}
\end{gathered}
$$

where $\mathbf{l}_{0}=\left(\mathbf{v}_{A}-\mathbf{v}_{E}\right) \times\left(\mathbf{v}_{B}-\mathbf{v}_{D}\right), \mathbf{l}_{1}=\left(\mathbf{v}_{B}-\mathbf{v}_{D}\right) \times\left(\mathbf{v}_{E}-\mathbf{v}_{C}\right)$ and $\mathbf{l}_{2}=\left(\mathbf{v}_{D}-\mathbf{v}_{A}\right) \times\left(\mathbf{v}_{C}-\mathbf{v}_{E}\right)$. Since all of the quantities involved in Eqs. (17)-(19) are explicitly given, we do not provide their lengthy forms here.

Furthermore, there are two additional nonoverlapping conditions given by 4 potential vertex-to-face contacts, i.e.,

$$
\boldsymbol{\lambda}_{2} \cdot \mathbf{n}_{1} \geq \sqrt{\frac{2}{3}}
$$

where $\mathbf{n}_{1}=\left(\frac{\sqrt{6}}{3},-\frac{2 \sqrt{2}}{6},-\frac{1}{3}\right)$ is the unit outward normal of the contacting face,

$$
\left(\boldsymbol{\lambda}_{1}-\boldsymbol{\lambda}_{2}\right) \cdot \mathbf{n}_{2} \geq \sqrt{\frac{2}{3}},
$$

and $\mathbf{n}_{2}=\left(-\frac{\sqrt{6}}{3},-\frac{2 \sqrt{2}}{6}, \frac{1}{3}\right)$ is the unit outward normal of the contacting face. The edge-toedge and vertex-to-face contacts are realized when the equality holds in the above conditions (15)-(21). Note we do not provide the lengthy forms of the above equations because the quantities involved have been given above. These nonoverlapping conditions constrain the possible values of the remaining three variables.

Finally, the density of the six-parameter packings is given by

$$
\phi=\frac{4 V_{T}}{V_{F}}=\frac{4 V_{T}}{\left|\boldsymbol{\lambda}_{1} \times \boldsymbol{\lambda}_{2} \cdot \boldsymbol{\lambda}_{3}\right|},
$$

where $V_{T}=\sqrt{2} / 12$ is the volume of a regular tetrahedron with unit edge length and $V_{F}$ is the volume of the fundamental cell given by 


$$
\begin{aligned}
V_{F}= & \frac{1}{3}\left(\alpha_{2} \beta_{1}+\alpha_{1} \beta_{2}\right)\left(-4 \sqrt{6}+\sqrt{6} \alpha_{1}+3 \sqrt{2} \alpha_{2}-2 \alpha_{3}+2 \sqrt{6} \beta_{1}+\beta_{3}\right) \\
& -\frac{1}{12}\left(\alpha_{3} \beta_{1}+\alpha_{1} \beta_{3}\right)\left(-2 \sqrt{3} \alpha_{1}-10 \alpha_{2}+3 \sqrt{2} \alpha_{3}+8 \beta_{2}\right) \\
+ & \frac{1}{12}\left(-\alpha_{3} \beta_{2}+\alpha_{2} \beta_{3}\right)\left(-6 \alpha_{1}-2 \sqrt{3} \alpha_{2}+\sqrt{6} \alpha_{3}-2 \sqrt{6} \beta_{3}\right) .
\end{aligned}
$$

The packing density relation (22) in conjunction with (23) is a function of the 6 variables $\alpha_{i}$ and $\beta_{i}(i=1,2,3)$. We optimize the density for this problem using a sequential linear programming method subject to the nonoverlapping constraints given by Eqs. (15)-(21) that specify all of the remaining possible contacts. In this way, we obtain the densest known packings with density $\phi=0.856347 \ldots$ This is a numerical verification of the optimality of the these packings among all four-particle basis packings. In the following section, we provide analytical constructions of the densest known packings and other dense packings from our general six-parameter family.

\section{B. Three-Parameter Family of Dense Tetrahedron Packings}

Both our simulations and local numerical analysis of Eqs. (22) and (23) suggest that realizing the face-to-face contacts associated with Eqs. (15) and (16) and the edge-to-edge contact associated with Eq. (17) will lead to denser packings. Therefore, we let the equality hold in Eqs. (15), (16) and (17). By solving these three additional equations, we can further eliminate three independent variables for the packing, i.e.,

$$
\begin{gathered}
\alpha_{2}=\sqrt{3} / 2 \\
\beta_{2}=\frac{1}{4}\left(-2 \sqrt{3} \alpha_{1}+2 \alpha_{2}+3 \sqrt{2} \alpha_{3}\right), \\
\beta_{3}=\frac{1}{10}\left(-8 \sqrt{6}+5 \sqrt{6} \alpha_{1}+3 \sqrt{2} \alpha_{2}+5 \sqrt{2} \alpha_{3}+10 \sqrt{6} \beta_{1}\right) .
\end{gathered}
$$

Therefore, the lattice vectors and the centroids of the dimers are functions of the three 
parameters $\left(\alpha_{1}, \alpha_{3}, \beta_{1}\right)$ only, i.e.,

$$
\begin{aligned}
& \mathbf{c}_{1}=\left(\frac{1}{5}-\frac{\alpha_{3}}{\sqrt{6}},-\frac{4}{5 \sqrt{3}},-\frac{\sqrt{6}}{5}-\alpha_{3}\right) \\
& \boldsymbol{\lambda}_{1}=\left(-\alpha_{1},-\frac{\sqrt{3}}{2},-\alpha_{3}\right) \\
& \boldsymbol{\lambda}_{2}=\left(\beta_{1},-\frac{\sqrt{3}}{4}+\frac{\sqrt{3}}{2} \alpha_{1}-\frac{3 \sqrt{2}}{4} \alpha_{3}, \frac{13 \sqrt{6}}{20}-\frac{\sqrt{6}}{2} \alpha_{1}-\frac{1}{2} \alpha_{3}-\sqrt{6} \beta_{1}\right), \\
& \boldsymbol{\lambda}_{3}=\left(\frac{2}{5}-\alpha_{1}-\beta_{1},-\frac{\sqrt{3}}{4}-\frac{\sqrt{3}}{2} \alpha_{1}+\frac{3 \sqrt{2}}{4} \alpha_{3},-\frac{21 \sqrt{6}}{20}+\frac{\sqrt{6}}{2} \alpha_{1}-\frac{1}{2} \alpha_{3}+\sqrt{6} \beta_{1}\right) .
\end{aligned}
$$

The density of the packing is given by

$$
\phi=\frac{4 V_{T}}{V_{F}}=\frac{\sqrt{2}}{3 V_{F}},
$$

where $V_{F}$ is the volume of the fundamental cell given by

$$
V_{F}=\frac{1}{100}\left(39 \sqrt{2}-60 \sqrt{2} \alpha_{1}^{2}+40 \sqrt{3} \alpha_{1} \alpha_{3}+30 \sqrt{2} \alpha_{3}^{2}\right)
$$

Note that although the packing structures depend on all three variables $\left(\alpha_{1}, \alpha_{3}, \beta_{1}\right)$, the density is only dependent on two variables $\left(\alpha_{1}, \alpha_{3}\right)$. The feasible values of the remaining variables are determined by Eqs. (17)-(21). In particular, we have

$$
\begin{gathered}
\frac{3}{2} \alpha_{1}-\frac{5 \sqrt{6}}{12} \alpha_{3} \leq \frac{1}{20}, \\
\frac{3}{2} \alpha_{1}-\frac{5 \sqrt{6}}{12} \alpha_{3} \geq-\frac{1}{20}, \\
\max \left\{\frac{7}{10}-\alpha_{1}+\frac{\sqrt{6}}{6} \alpha_{3}, \frac{7}{10}-\frac{\sqrt{6}}{6} \alpha_{3}\right\} \leq \beta_{1} \leq \min \left\{\frac{3}{4}+\frac{1}{2} \alpha_{1}-\frac{\sqrt{6}}{4} \alpha_{3}, \frac{3}{4}-\frac{3}{2} \alpha_{1}+\frac{\sqrt{6}}{4} \alpha_{3}\right\} .
\end{gathered}
$$

From Eq. (28), we see that maximizing the density is equivalent to minimizing the volume of the fundamental cell $V_{F}$. To obtain our two-parameter family of packings reported in Ref. [36], we assume that the minimum of $V_{F}$ can be obtained by optimizing the two variables $\left(\alpha_{1}, \alpha_{3}\right)$ separately, i.e., we assume the following is true:

$$
\min _{\left\{\forall\left(\alpha_{1}, \alpha_{3}\right)\right\}} V_{F}=\min _{\left\{\forall \alpha_{1}\right\}}\left[\min _{\left\{\forall \alpha_{3}\right\}} V_{F}\right]
$$




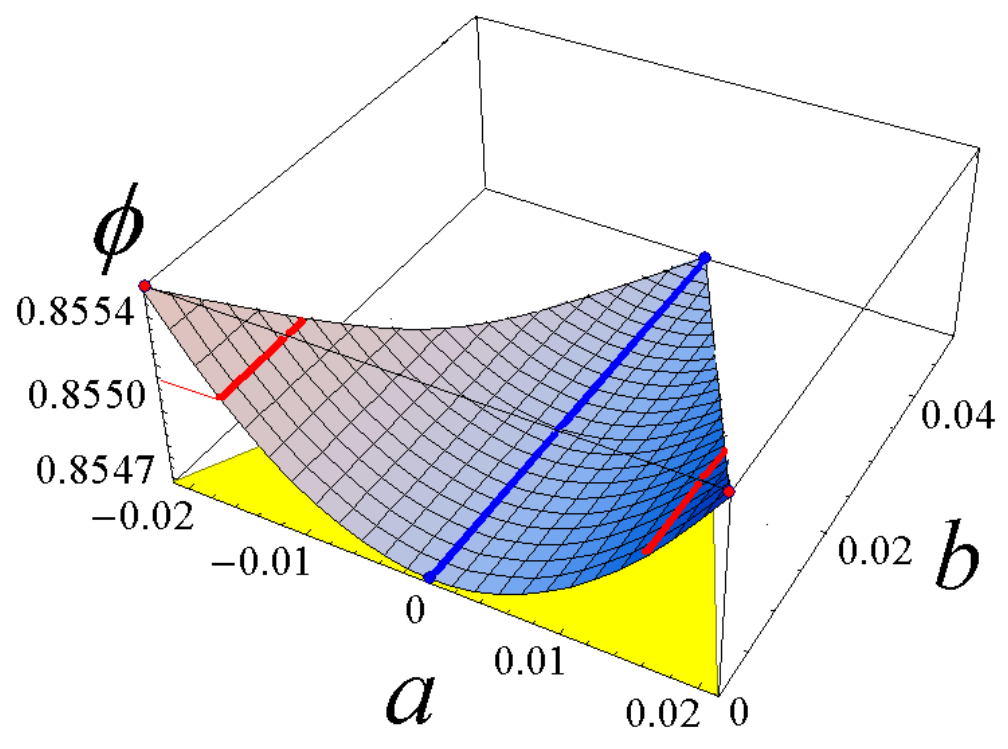

FIG. 2: (color online). The density $\phi$ surface of our family of tetrahedron packings as a function of the two parameters $a$ and $b$ (the parameters are related to the original variables via $\alpha_{1}=a$, $\alpha_{3}=-\sqrt{\frac{2}{3}} a, \beta_{1}=\frac{3}{4}-2 a-b$ for $\alpha_{1}>0$ and $\alpha_{1}=a, \alpha_{3}=-\sqrt{\frac{2}{3}} a, \beta_{1}=\frac{3}{4}+a-b$ for $\left.\alpha_{1}<0\right)$. As explained in the text, the thick red lines (associated with $a \approx \pm 0.016$ ) show two sets of tetrahedron packings with distinct structures but with the same density. The two red points (associated with $\left.a= \pm \frac{3}{140}\right)$ correspond to the densest two tetrahedron packings in this family. The packings found by Kallus et al. 35] are recovered from our two-parameter family (thick blue line, associated with $a=0)$.

The inner optimization (over $\alpha_{3}$ with fixed $\alpha_{1}$ ) requires

$$
\partial V_{F} / \partial \alpha_{3}=\frac{1}{5}\left(2 \sqrt{3} \alpha_{1}+3 \sqrt{2} \alpha_{3}\right)=0
$$

which gives $\alpha_{3}=-\sqrt{\frac{2}{3}} \alpha_{1}$. This further reduces the number of variables and leads to a two-parameter family of packings. Substituting this expression into Eq. (29), we obtain

$$
V_{F}=\frac{1}{100}\left(39 \sqrt{2}-80 \sqrt{2} \alpha_{1}^{2}\right) .
$$

Substituting Eq. (33) into Eq. (28) leads to the density expression

$$
\phi=\frac{100}{117-240 \alpha_{1}^{2}} .
$$

where $\alpha_{1} \in\left(-\frac{3}{140}, \frac{3}{140}\right)$. It is important to note that for each $\alpha_{1} \neq 0$, there are two sets of packings of tetrahedra, each with distinct structures but possessing the same density (as 
shown in Fig. 2 by the thick red lines). In addition, by substituting $\alpha_{1}=a, \alpha_{3}=-\sqrt{\frac{2}{3}} a$, $\beta_{1}=\frac{3}{4}-2 a-b$ for $\alpha_{1}>0$ and $\alpha_{1}=a, \alpha_{3}=-\sqrt{\frac{2}{3}} a, \beta_{1}=\frac{3}{4}+a-b$ for $\alpha_{1}<0$, respectively into Eq. (27), the two sets of lattice vectors and dimer centroids can be obtained, i.e., for $-\frac{3}{140}<a<0$, we have

$$
\begin{gathered}
\mathbf{c}_{1}=\left(\frac{1}{5}+\frac{a}{3},-\frac{4}{5 \sqrt{3}},-\frac{3 \sqrt{2}}{5 \sqrt{3}}+\sqrt{\frac{2}{3}} a\right), \\
\boldsymbol{\lambda}_{1}=\left(-a,-\frac{\sqrt{3}}{2}, \sqrt{\frac{2}{3}} a\right), \\
\boldsymbol{\lambda}_{2}=\left(\frac{3}{4}+a-b,-\frac{\sqrt{3}}{4}+\sqrt{3} a,-\frac{3}{5 \sqrt{6}}-\frac{8}{\sqrt{6}} a+\sqrt{6} b\right), \\
\boldsymbol{\lambda}_{3}=\left(-\frac{7}{20}-2 a+b,-\frac{\sqrt{3}}{4}-\sqrt{3} a,-\frac{9}{5 \sqrt{6}}+\frac{10}{\sqrt{6}} a-\sqrt{6} b\right),
\end{gathered}
$$

where $0<b<\frac{3+140 a}{60}$; for $0<a<\frac{3}{140}$, we have

$$
\begin{gathered}
\mathbf{c}_{1}=\left(\frac{1}{5}+\frac{a}{3},-\frac{4}{5 \sqrt{3}},-\frac{3 \sqrt{2}}{5 \sqrt{3}}+\sqrt{\frac{2}{3}} a\right), \\
\boldsymbol{\lambda}_{1}=\left(-a,-\frac{\sqrt{3}}{2}, \sqrt{\frac{2}{3}} a\right), \\
\lambda_{2}=\left(\frac{3}{4}-2 a-b,-\frac{\sqrt{3}}{4}+\sqrt{3} a,-\frac{3}{5 \sqrt{6}}+\frac{10}{\sqrt{6}} a+\sqrt{6} b\right), \\
\lambda_{3}=\left(-\frac{7}{20}+a+b,-\frac{\sqrt{3}}{4}-\sqrt{3} a,-\frac{9}{5 \sqrt{6}}-\frac{8}{\sqrt{6}} a-\sqrt{6} b\right),
\end{gathered}
$$

where $0<b<\frac{3-140 a}{60}$.

The densest packings in this family are associated with $a=-\frac{3}{140}, b=0$ and $a=\frac{3}{140}$, $b=0$, possessing a density $\phi_{\max }=\frac{12250}{14319}=0.855506 \ldots$ In each set, there is a unique packing structure associated with $\phi_{\max }$ (shown as the red points in Fig. 21), instead of a spectrum of structures. Two different configurations of the densest packings of the two-parameter family within their corresponding rhombohedral fundamental cells are shown in Fig. 3(a) and (b). The two four-particle configurations in the fundamental cells are only slightly different from one another. Specifically, the difference between the coordinates of the dimer centroids that are not at the origin (i.e., at $\mathbf{c}_{1}$ ) is $\left(\frac{1}{70}, 0, \frac{\sqrt{6}}{70}\right)$. Figure $4(a)$ and (b) depict periodic replicates of the densest tetrahedral packings corresponding to those shown in Fig. 3(a) and (b) with 8 fundamental cells ( 2 along each lattice vector). These packings are not chiral. The lattice vectors of packings associated with positive and negative $a$ are related by an isometric map [37]. 


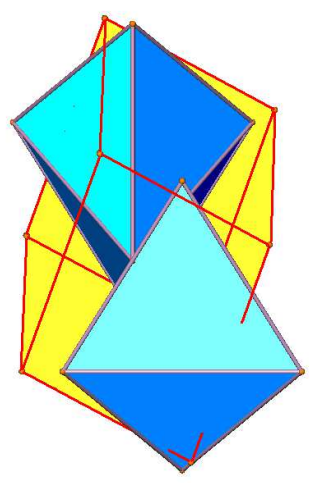

(a)

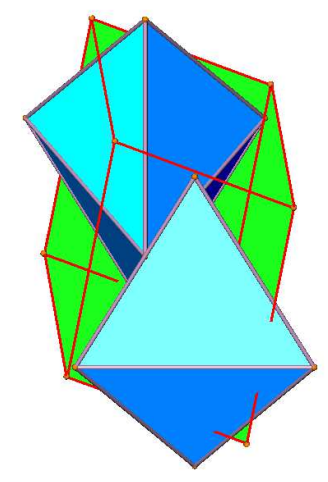

(c)

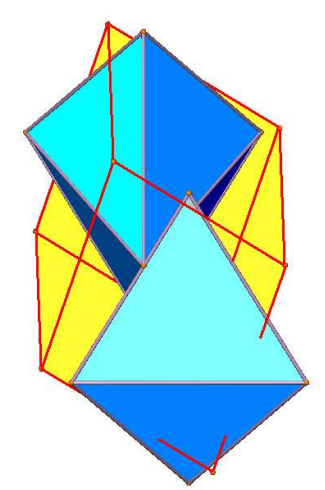

(b)

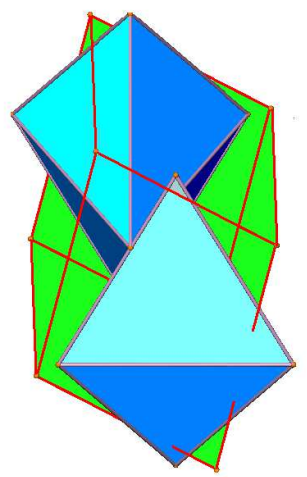

(d)

FIG. 3: (color online). In the top panel [(a) and (b)], we show the two different configurations of the densest packings of four tetrahedra (two dimers) with $\phi=\frac{12250}{14319}=0.855506 \ldots$ within their corresponding rhombohedral fundamental cells of the two-parameter family. (a) Here $a=-\frac{3}{140}$ and $b=0$. (b) Here $a=\frac{3}{140}$ and $b=0$. The fundamental cells are colored yellow (light gray) and their boundaries are colored red (dark gray). The two packings and their associated fundamental cells shown in (a) and (b) are only slightly different from one another. In the bottom panel [(c) and $(\mathrm{d})$ ], we show the two different configurations of the densest packings of four tetrahedra (two dimers) with $\phi=\frac{4000}{4671}=0.856347 \ldots$ within their corresponding rhombohedral fundamental cells of the three-parameter family. (c) Here $\alpha_{1}=\frac{7}{160}, \alpha_{3}=\frac{3}{160} \sqrt{\frac{2}{3}}$ and $\beta_{1}=\frac{111}{160}$. (d) Here $\alpha_{1}=-\frac{7}{160}$, $\alpha_{3}=-\frac{3}{160} \sqrt{\frac{2}{3}}$ and $\beta_{1}=\frac{59}{80}$. The fundamental cells are colored green (medium gray) and their boundaries are colored red (dark gray). The two packings and their associated fundamental cells shown in (c) and (d) are only slightly different from one another. 


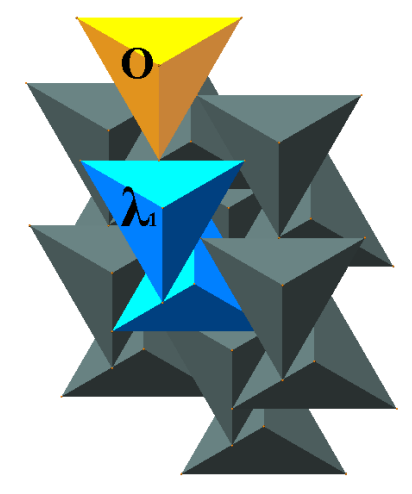

(a)

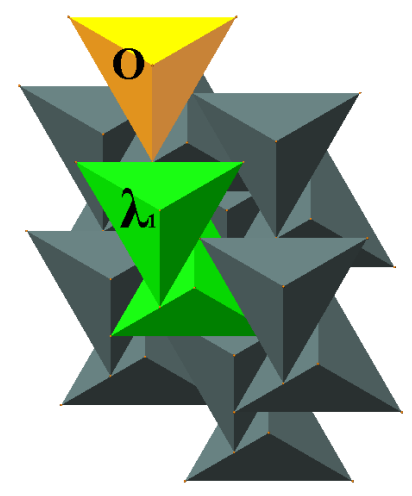

(c)

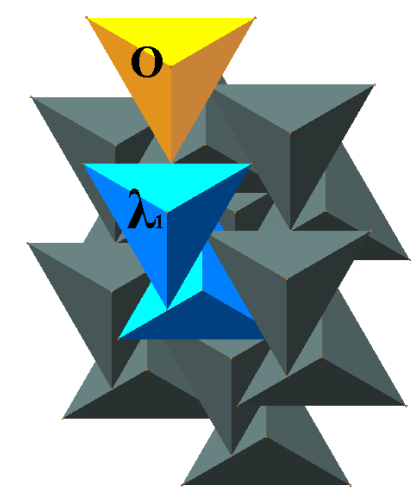

(b)

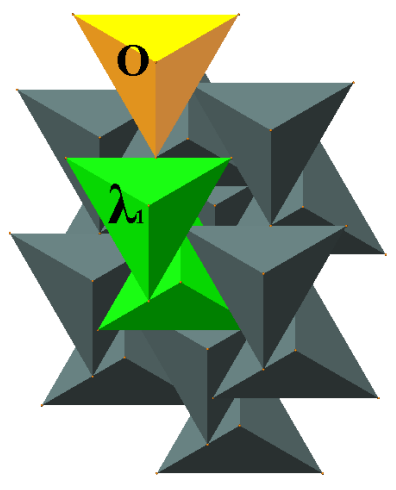

(d)

FIG. 4: (color online). This figure shows periodic replicates the densest tetrahedron packings of the two-parameter family (with $\phi=\frac{12250}{14319}=0.855506 \ldots$ ) and the three-parameter family ( with $\left.\phi=\frac{4000}{4671}=0.856347 \ldots\right)$ corresponding to the top and bottom panels in Fig. 3, respectively, with 8 fundamental cells (2 along each lattice vector). The tetrahedra within the fundamental cells are shown in blue and green (both appearing as medium gray in the print version) for the two-parameter [(a) and (b)] and three-parameter [(c) and (d)] family packings, respectively. Note that in (a), the dimer with centroid at $\boldsymbol{\lambda}_{1}$ (blue, or medium gray in the print version) is slightly shifted to the right with respect to the dimer at the origin $\mathbf{O}$ (yellow, or light gray in the print version); and in (b), the dimer with the centroid at $\boldsymbol{\lambda}_{1}$ (blue, or medium gray in the print version) is slightly shifted to the left with respect to the dimer at the origin $\mathbf{O}$ (yellow, or light gray in the print version). The three-parameter packing structures are very similar to that of the twoparameter packings. Observe that in the perspective shown the dimers appear as if they were single tetrahedra. 
At $a=0$, there is only one set of packings with the same $\phi=\frac{100}{117}=0.85470 \ldots$, whose structures are dependent on $b$ (shown as the blue line in Fig. 21). These packings reduce exactly to those discovered by Kallus et al., which possess relatively high symmetry. In particular, $a=0$ allows the centroids of the dimers, which are related to each other by an integer multiple of $\boldsymbol{\lambda}_{1}$, to be perfectly aligned on two of the mirror image planes of the dimers simultaneously, which leads to additional two-fold rotational symmetry of the packing. This additional rotational symmetry, together with the point inversion symmetry, leads to uniform packings with respect to each tetrahedron (not just each dimer), i.e., the symmetry operation acts to take each tetrahedron into another.

If we do not make the assumption used above (i.e., optimizing the two variables $\alpha_{1}$ and $\alpha_{3}$ separately), the boundary of the domain of $V_{F}$ needs to be searched completely to find the minimal value. For the boundary specified by

$$
\frac{3}{2} \alpha_{1}-\frac{5 \sqrt{6}}{12} \alpha_{3} \leq \frac{1}{20}
$$

we have $\alpha_{1}=\frac{1}{90}\left(3+25 \sqrt{6} \alpha_{3}\right)$. Substituting this expression into Eq. (29) gives

$$
V_{F}=\frac{2}{1125}\left(219 \sqrt{2}-5 \sqrt{3} \alpha_{3}+200 \sqrt{2} \alpha_{3}^{2}\right)
$$

the minimal value of which is $V_{F}^{*}=\frac{1557}{2000 \sqrt{2}}$ with $\alpha_{3}^{*}=\frac{3}{160} \sqrt{\frac{2}{3}}$. For the boundary specified by

$$
\frac{3}{2} \alpha_{1}-\frac{5 \sqrt{6}}{12} \alpha_{3} \geq-\frac{1}{20}
$$

we have $\alpha_{1}=\frac{1}{90}\left(-3+25 \sqrt{6} \alpha_{3}\right)$. Substituting this expression into Eq. (29) gives

$$
V_{F}=\frac{2}{1125}\left(219 \sqrt{2}+5 \sqrt{3} \alpha_{3}+200 \sqrt{2} \alpha_{3}^{2}\right)
$$

the minimal value of which is $V_{F}^{*}=\frac{1557}{2000 \sqrt{2}}$ with $\alpha_{3}^{*}=-\frac{3}{160} \sqrt{\frac{2}{3}}$.

Thus, we find a maximal density $\phi_{\max }=\frac{4000}{4671}$, which was also reported by Chen et al. [37], associated with $\alpha_{1}=\frac{7}{160}, \alpha_{3}=\frac{3}{160} \sqrt{\frac{2}{3}}$ and $\alpha_{1}=-\frac{7}{160}, \alpha_{3}=-\frac{3}{160} \sqrt{\frac{2}{3}}$. The packing structures can be obtained by substituting $\beta_{1}=\frac{3}{4}-\frac{3}{2} \alpha_{1}+\frac{\sqrt{6}}{4} \alpha_{3}=\frac{111}{160}$ for $\alpha_{1}>0$ and $\beta_{1}=\frac{3}{4}+\frac{1}{2} \alpha_{1}-\frac{\sqrt{6}}{4} \alpha_{3}=\frac{59}{80}$ for $\alpha_{1}<0$ into Eq. (27). These three-parameter packings are very close to the two-parameter packings in structure and possess the same symmetry [see Fig. 3(c) and (d), and Fig. 4(c) and (d)]. 


\section{TOWARDS UPPER BOUNDS ON THE MAXIMAL DENSITY}

The problem of determining upper bounds on the maximal density of packings of nonspherical particles is highly nontrivial, and yet such estimates would be indispensable in assessing the packing efficiency of a candidate dense packing, especially if tight upper bounds could be constructed. It has recently been shown that $\phi_{\max }$ of a packing of congruent nonspherical particles of volume $v_{P}$ in $\mathbb{R}^{3}$ is bounded from above according to

$$
\phi_{\max } \leq \min \left[\frac{v_{P}}{v_{S}} \frac{\pi}{\sqrt{18}}, 1\right],
$$

where $v_{S}$ is the volume of the largest sphere that can be inscribed in the nonspherical particle and $\pi / \sqrt{18}$ is the maximal sphere-packing density [28, 29]. The upper bound (41) will be relatively tight for packings of nonspherical particles provided that the asphericity $\gamma$ (equal to the ratio of the circumradius to the inradius) of the particle is not large. However, for tetrahedra, the asphericity is too large for the upper bound (41) to yield a result that is less than unity.

One possible approach to obtaining nontrivial upper bounds is to attempt to generalize the idea that Rogers used to prove upper bounds on $\phi_{\max }$ for sphere packings [38]. The key concept is to consider a locally dense cluster of 4 contacting spheres in a tetrahedral arrangement and then prove that the fraction of space covered by the spheres within the tetrahedron joining the sphere centers is an upper bound on $\phi_{\max }$. This can be done because one can triangulate any sphere packing to decompose it into generally irregular tetrahedra with vertices at sphere centers. The fact that the regular tetrahedron has the best density for any tetrahedron, then yields an upper bound for the density of any sphere packing. In the case of the non-tiling Platonic and Archimedean solids, a natural choice for the enclosing region associated with the cluster is its convex hull.

For tetrahedra, we must identify the densest local cluster with density that exceeds or equals $\phi_{\max }$ while taking into account their shape symmetry. A trivial choice is a dimer because the fraction of space covered by the dimer within its convex hull is unity. Two nontrivial choices are the 5-particle "wagon-wheel" cluster as shown in Fig. 5(a) and a more "isotropic" icosahedron-like cluster (a cluster consisting of 20 tetrahedra that share a common vertex) as shown in Fig. [5(b). The "wagon-wheel" and icosahedron-like cluster can be considered to be the most anisotropic and isotropic densest local clusters, respectively. The former is essentially a "flat" (quasi-two-dimensional) structure. Since neither of the 

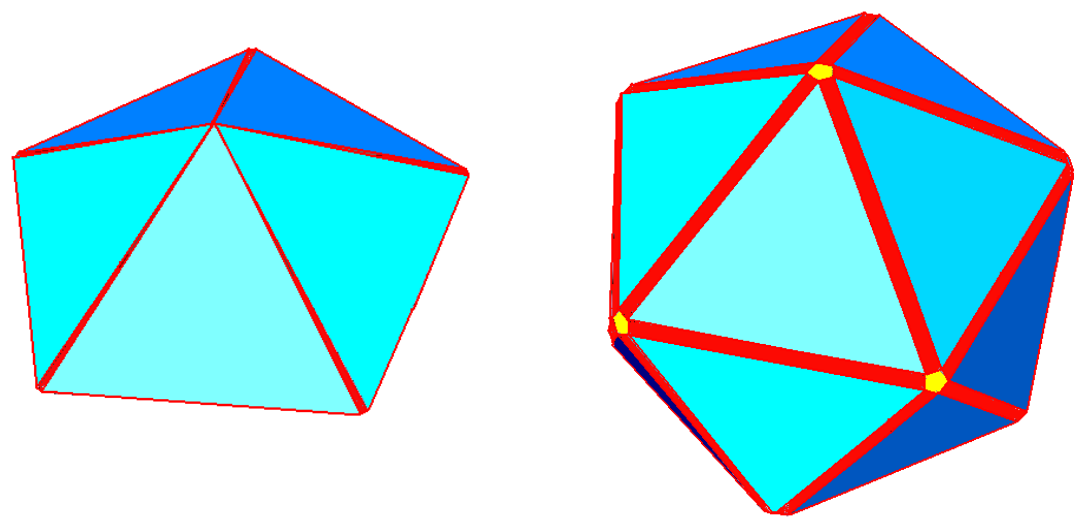

(a)

(b)

FIG. 5: (color online). (a) The convex hull of five regular tetrahedra in a "wagon wheel" arrangement. The convex hull can be decomposed into five regular tetrahedra (shown in blue, or medium gray in the print version) and five thin irregular tetrahedra (shown in red, or dark gray in the print version). (b)The convex hull of 20 regular tetrahedra in an "icosahedral" arrangement. The convex hull can be decomposed into 20 regular tetrahedra (shown in blue, or medium gray in the print version), 12 pyramids with pentagonal bases (shown in yellow, or light gray in the print version) and 30 pyramid with rectangular bases (shown in red, or dark gray in the print version).

two local clusters can tile space, their local packing densities, which are computed below, provide limiting estimates on upper bounds for $\phi_{\max }$.

The convex hull of the "wagon-wheel" cluster composed of five tetrahedra sharing a common edge (a "wagon wheel" cluster) can be decomposed into five regular tetrahedra and five thin irregular tetrahedra. We assume the gaps between the regular tetrahedra are equal, i.e, the thin irregular tetrahedra are congruent. Note the regular tetrahedron shares two faces with its neighboring irregular tetrahedra. Thus, the volume ratio is equal to the ratio of the corresponding heights of the regular and irregular tetrahedron, i.e.,

$$
\gamma=\frac{V_{T}}{V_{T_{*}}}=\frac{1}{\frac{\sqrt{2}}{2}\left(3 \cos ^{2} \frac{3 \pi}{10}-1\right)},
$$

where $V_{T}$ and $V_{T_{*}}$ is the volume of the regular and irregular tetrahedron, respectively. Thus, the density of this local packing, defined as the fraction space covered by the regular tetra- 
hedra within the convex hull is given by

$$
\phi_{W}=\frac{V_{T}}{V_{T}+V_{T_{*}}}=\frac{1}{\frac{3 \sqrt{2}}{2} \cos ^{2} \frac{3 \pi}{10}+\left(1-\frac{\sqrt{2}}{2}\right)}=0.974857 \ldots
$$

Because this cluster is highly anisotropic and is an effectively "flat" object, it is reasonable to assume that it is not the least densest local cluster and therefore its local density is likely to be gross overestimate of the best upper bound on $\phi_{\max }$.

It seems reasonable to suppose that the least densest local cluster with the highest shape symmetry is one consisting of 20 tetrahedra sharing a common vertex, i.e., an icosahedronlike cluster. The convex hull of such a cluster can be decomposed into 20 regular tetrahedra, 12 pyramids with pentagonal bases and 30 pyramid with rectangular bases [see Fig. 5(b)]. We assume the gaps between tetrahedra are equal and thus the two types of pyramids are congruent. The volume of the regular tetrahedron is $V_{T}=\frac{\sqrt{2}}{12}$ and the volume $V_{P}$ of the pyramid with pentagonal base is given by

$$
V_{P}=\frac{5}{12} \tan \left(\frac{3 \pi}{10}\right) L^{2} \sqrt{1-\frac{L^{2}}{4 \cos ^{2} \frac{3 \pi}{10}}},
$$

and the volume $V_{R}$ of the pyramid with rectangular base is given by

$$
V_{R}=\frac{\sqrt{2}}{6} L \sqrt{1-L^{2}}
$$

where

$$
L=\left(\frac{2 \sqrt{2}}{\tau^{2}}-1\right) \sqrt{\frac{1}{6}+\frac{\sqrt{5}}{18}}
$$

and $\tau=(1+\sqrt{5}) / 2$ is the golden ratio. Thus, the local packing density is given by

$$
\phi_{I}=\frac{20 V_{T}}{20 V_{T}+12 V_{P}+30 V_{R}}=0.880755 \ldots
$$

Note in the above calculations, we have assumed that the gaps between the tetrahedra are equal, which is sufficient to provide an estimate of the fraction of space covered by the cluster within its convex hull. It is noteworthy that the icosahedron-like cluster of 20 tetrahedra is at least a local extremum, i.e., adding or removing a tetrahedron from the cluster will decrease the local packing density. Moreover, adding another "shell" of tetrahedra around the icosahedron-like cluster will also reduce the local packing density. We note that the idea 
of Rogers to prove an upper bound for sphere packings cannot be used here because there is no analogous decomposition of space into irregular convex hulls shown in Fig. 5(a) or (b). A completely new idea is needed to prove that the aforementioned estimates are bounds. If one could prove that these clusters are indeed locally denser than the globally densest packings, then the aforementioned estimates provide upper bounds on $\phi_{\max }$, but they cannot be sharp, i.e., they are not achievable by any packings. Without loss of generality, it is reasonable to expect that the globally densest packings of tetrahedra contain a particular proportion of both the isotropic and anisotropic local clusters. Therefore, these limiting estimates provide a plausible range of upper bounds, i.e., $\phi_{\max }^{U} \in[0.880755,0.974857]$. It is noteworthy that the density $\phi=0.856347 \ldots$ of the densest known packings of tetrahedra is relatively close to this putative lower-limit upper bound density estimate of $0.880755 \ldots$...

\section{CONCLUSIONS AND DISCUSSION}

For all of the small periodic packings that we investigated (including 2 to 32 particles per fundamental cell) using our numerical ASC scheme, the densest packings that emerged had a 4-particle basis. The numerical optimization that we performed of our six-parameter family of tetrahedron packings verified the optimality of the densest 4-particle packing with density $\phi=\frac{4000}{4671}=0.856347 \ldots$... Are these dimer packings optimal among all packings? This is a difficult question to answer in any definitive way. We first note that the two contacting dimers in the fundamental cell possess center inversion symmetry and thus they can be viewed as a centrally symmetric compound object. Hence, our two-parameter family of packings for the two-dimers (and the densest known packings) are Bravais lattice packings of such compound objects. Thus, it is not very surprising that dense Bravais lattice packings of this centrally symmetric compound object have a fairly high density based on the arguments leading to Conjectures 1 and 2 [28, 29], i.e., there are a large number of face-to-face contacts which are made possible due to the central symmetry of the compound object and bring the centroids of the objects closer to each other. However, Conjecture 3 states that central symmetry alone does not guarantee optimality of the densest Bravais lattice packings among all packings. That is, there are additional constraints on the geometry and shape of the objects, e.g., they might also need to possess three equivalent principal axes, as do the centrally symmetric Platonic and Archimedean solids. When the latter condition does not hold, 
there could exist non-Bravais lattice packings that are denser than the optimal lattice packings (e.g., ellipsoids [21]). The 4-tetrahedron compound object is very anisotropic and does not possess equivalent principal axes. Moreover, it is a concave object rather than convex, which makes it even more difficult to make a definitive conclusion about the optimality of the densest dimer packings. In addition, Haji-Akbari et al. [34] have found periodic packings of tetrahedra consisting of 82 particles per fundamental cell with a slightly lower density (i.e., $\phi \approx 0.8503)$ than that of the densest dimer packings. Their packings possess much larger fundamental cells with distinctly different and more complicated particle arrangements than the dimer packings. This suggests that there is a large degeneracy of different non-Bravais lattice packing structures with high densities with various levels of complexity and perhaps even higher densities than the optimal 4-particle packings. Such denser packings could be discovered by carrying out exhaustive searches to determine the globally maximal densities of packings with successively larger numbers of particles per fundamental cell.

Previous numerical studies have indicated that dense packings may have a large number of particles per fundamental cell arranged in a complex fashion, e.g., the "disordered wagonwheels" packing with $\phi=0.822637 \ldots$. 29] and the "ring stacks" packing with $\phi=0.8503 \ldots$ [34]. However, it is now clear that such packings are in fact only locally optimal solutions and hence the numerical techniques used to obtain them are incapable of extricating themselves from these "trapped" regions of configuration space to find denser and more ordered structures due to the intrinsic geometrical frustration of the tetrahedron mentioned earlier. This may also call into question claims made by Haji-Akbari et al. [34] that their packings, which are characterized by an effective "quasicrystal-like" plane, are true thermodynamic equilibrium phases of tetrahedra at high densities. Instead, our high-density constructions suggest that uniform periodic packings with a 4-particle basis (or even some yet unknown denser periodic packing) and their unjammmed, lower-density counterparts could be the stable phases at high densities. If the latter is correct and the putative "quasicrystal-like" phase truly exists at intermediate densities, then it is hard to imagine how such complex quasicrystal-like structures of tetrahedra under quasi-static compression (densification) could rearrange themselves at higher densities to spontaneously form a more ordered periodic arrangement with higher symmetry. However, it is difficult to draw any such definitive conclusions without further study.

Although there could still be tetrahedron packings denser than our constructions, it ap- 
pears that all of the evidence thus far points to the fact that the densest tetrahedron packings cannot possess very high symmetry [28 30,33, 34] due to the lack of central symmetry of a tetrahedron and because tetrahedra cannot tile space [29]. Indeed, the dense 4-particle-basis packings found in the present paper improved upon the best dimer packings of Kallus et al. [35] by relaxing the two-fold rotational symmetry constraints they imposed.

\section{Acknowledgments}

We are grateful to Henry Cohn, Yoav Kallus and John Conway for helpful discussions. S. T. thanks the Institute for Advanced Study for its hospitality during his stay there. This work was supported by the Division of Mathematical Sciences at the National Science Foundation under Award Number DMS-0804431 and by the MRSEC Program of the National Science Foundation under Award Number DMR-0820341.

[1] J. D. Weeks, D. Chandler, and H. C. Andersen, J. Chem. Phys. 54, 5237 (1971).

[2] R. Zallen, The Physics of Amorphous Solids (Wiley, New York, 1983).

[3] P. M. Chaikin and T. C. Lubensky, Principles of Condensed Matter Physics (Cambridge University Press, New York, 1995).

[4] S. Torquato, Random Heterogeneous Materials: Microstructure and Macroscopic Properties (Springer-Verlag, New York, 2002).

[5] C. F. Gauss, Göttingsche Gelehrte Anzeigen (1831), see also J. reine angew. Math., vol. 20, $1840,312-320$.

[6] J. D. Bernal, Nature 185, 68 (1960).

[7] J. H. Conway and N. J. A. Sloane, Sphere Packings, Lattices and Groups (Springer-Verlag, New York, 1998).

[8] A. J. Liu and S. R. Nagel, Nature 396, 21 (1998).

[9] S. Torquato, T. M. Truskett, and P. G. Debenedetti, Phys. Rev. Lett. 84, 2064 (2000).

[10] C. S. O'Hern, S. A. Langer, A. J. Liu, and S. R. Nagel, Phys. Rev. Lett. 88, 075507 (2002).

[11] H. Cohn and N. Elkies, Annals Math. 157, 689 (2003).

[12] T. C. Hales, Ann. Math. 162, 1065 (2005). 
[13] S. Torquato and F. H. Stillinger, Experimental Math. 15, 307 (2006).

[14] H. Cohn and A. Kumar, Ann. Math. 170, 1003 (2009).

[15] A. Donev, I. Cisse, D. Sachs, E. A. Variano, F. H. Stillinger, R. Connelly, S. Torquato, and P. M. Chaikin, Science, 303, 990 (2004).

[16] A. Donev, R. Connelly, F. H. Stillinger and S. Torquato, Phys. Rev. E, 75, 051304 (2007).

[17] M. Mailman, C. F. Schreck, C. S. O'Hern and B. Chakraborty, Phys. Rev. Lett. 102, 255501 (2009).

[18] Z. Zeravcic, N. Xu, A. J. Liu, S. R. Nagel and W. van Saarloos, Euro. Phys. Lett. 87, 26001 (2009).

[19] Y. Jiao, F. H. Stillinger and S. Torquato, Phys. Rev. E 81, 041304 (2010).

[20] U. Betke and M. Henk, Comput. Geom. 16, 157 (2000).

[21] A. Donev, F. H. Stillinger, P. M. Chaikin, and S. Torquato, Phys. Rev. Lett. 92, 255506 (2004).

[22] Y. Jiao, F. H. Stillinger, and S. Torquato, Phys. Rev. E 79, 041309 (2009).

[23] M. P. Allen, Phil. Trans. R. Soc. Lond. A 344, 323 (1993).

[24] D. Frenkel and J. F. Maguire, Mol. Phys. 49, 503 (1983).

[25] D. Frenkel, B. M. Mulder and J. P. McTague, Phys. Rev. Lett. 52, 287 (1984).

[26] G. Yatsenko and K. S. Schweizer, Langmuir 24, 7474 (2008).

[27] H. Cohn, Nature 460, 801 (2009).

[28] S. Torquato and Y. Jiao, Nature 460, 876 (2009).

[29] S. Torquato and Y. Jiao, Phys. Rev. E 80, 041104 (2009); 81, 049908(E) (2010).

[30] J. H. Conway and S. Torquato, Proc. Nat. Acad. Sci. 103, 10612 (2006).

[31] D. J. Hoylman, Bull. Amer. Math. Soc. 76, 135 (1970).

[32] P. M. Chaikin, S. Wang, and A. Jaoshvili, in Am. Phys. Soc. March Meeting (2007).

[33] E. R. Chen, Discrete Comput. Geom. 40, 214 (2008).

[34] A. Haji-Akbari, M. Engel, A. S. Keys, X. Zheng, R. G. Petschek, P. Palffy-Muhoray, and S. C. Glotzer, Nature 462, 773 (2009).

[35] Y. Kallus, V. Elser, and S. Gravel (2009), arXiv:0910.5226.

[36] S. Torquato and Y. Jiao, (2009), arXiv:0912.4210.

[37] E. R Chen, M. Engel, and S. C. Glotzer (2010), arXiv:1001.0586.

[38] C. A. Rogers, Proc. Lond. Math. Soc. 8, 609 (1958). 